\title{
Designing multi-shelled metal oxides: towards high energy-density lithium-ion batteries
}

\author{
Heng-guo Wang ${ }^{1,2}$ and Xin-bo Zhang ${ }^{1 *}$
}

Lithium ion batteries (LIBs), which dominate today's portable electronic market, have been actively investigated as energy sources for hybrid and electric vehicles [1]. However, their energy density has still fallen short of the increasing and demanding requirements. Currently, the most of cathode materials can only reversibly accept one electron and deliver capacities lower than 200 $\mathrm{mA} \mathrm{h} \mathrm{g}^{-1}$. To obtain high-energy LIBs, the cathode materials with large capacities should be developed. There is a general consensus that further enhancements in capacity will require a transition from the currently used $\mathrm{Li}^{+}$-insertion/deinsertion compounds, which are limited to $1 \mathrm{e}^{-}$ per formula-unit, to new compounds that are based on multi-electron-reaction materials [2].

Vanadium oxide, $\mathrm{V}_{2} \mathrm{O}_{5}$, is believed to be an attractive cathode material for next-generation LIBs due to the following three aspects [3-5]. First, $\mathrm{V}_{2} \mathrm{O}_{5}$ can capture two or three electrons and thus enables a high theoretical capacity of 294 or $441 \mathrm{~mA} \mathrm{~h} \mathrm{~g}^{-1}$, respectively. Second, its typical layered crystal structure makes reversible $\mathrm{Li}^{+}$insertion and extraction quite feasible, thus shows a good reversibility. Third, it has the advantage of low cost and high abundance in the Earth's crust. Unfortunately, up to now, the application of $\mathrm{V}_{2} \mathrm{O}_{5}$ is still greatly hindered by its poor cycling stability, upon deep discharge and low $\mathrm{Li}^{+}$diffusion coefficient.

The effective way to solve these problems is to fabricate micro-/nanostructured materials, especially porous and hollow structures [6,7]. On one hand, these structured materials could generate the enlarged surface area, provide a greater number of surface $\mathrm{Li}^{+}$-storage sites and better access for electrolytes, and further shorten ion/electron transportation paths. On the other hand, pore structures and inner cavity can buffer the volume expansion and alleviate the stress and strain induced by the repeated $\mathrm{Li}^{+}$ insertion/extraction. Thus, continual researches have been devoted to fabricating such structured $\mathrm{V}_{2} \mathrm{O}_{5}$ electrode materials. However, only simple nanostructure could be easily prepared and constructing complex hollow structures still needs the synthesis of the sacrificial templates and additional step of etching templates, which is usually time consuming and costly. Furthermore, the cycling stability in these materials is not effectively enhanced, especially upon deep discharge in the voltage range of 4.0-1.5 $\mathrm{V}$ ( $v s$. $\left.\mathrm{Li} / \mathrm{Li}^{+}\right)$. Therefore, it is still a big challenge to develop a facile strategy to synthesize the hollow micro-/nanostructured $\mathrm{V}_{2} \mathrm{O}_{5}$ cathode materials with designed multilevel interior structures.

Recent work by Yu, Zhang and Wang et al. [8] has creatively combined the anion adsorption and catalytic combustion process to construct multi-shelled/cavitied $\mathrm{V}_{2} \mathrm{O}_{5}$ hollow microspheres $\left(\mathrm{M}-\mathrm{V}_{2} \mathrm{O}_{5}-\mathrm{HMs}\right)$ (Fig. 1a). In fact, the electrostatic attraction between negatively charged templates and metal cations has been successfully applied in the synthesis of M-HMs (Route $\mathrm{i}$ in Fig. 1a), but failed in synthesizing $\mathrm{M}-\mathrm{V}_{2} \mathrm{O}_{5}-\mathrm{HMs}$. Herein, the anion-adsorption approach could be adopted to continuously adsorb anions (mainly $\mathrm{VO}_{3}{ }^{-}$and $\mathrm{OH}^{-}$ions) and cations $\left(\mathrm{NH}_{4}{ }^{+}\right.$ion), and then generate $\mathrm{M}-\mathrm{V}_{2} \mathrm{O}_{5}-\mathrm{HMs}$ with the help of catalytic combustion process (Route ii in Fig. 1a). Benefitting from the unique structural properties, the prepared triple-shelled $\mathrm{V}_{2} \mathrm{O}_{5}$-HMs show exceptionally high specific capacities and outstanding cycling stability, even upon deep discharge in the voltage range of $4.0-1.5 \mathrm{~V}$ (Fig. 1b). All the obtained excellent electrochemical performances as well as the proposed strategy would open new avenue for developing

\footnotetext{
${ }^{1}$ State Key Laboratory of Rare Earth Resource Utilization, Changchun Institute of Applied Chemistry, Chinese Academy of Sciences, Changchun 130022, China

${ }^{2}$ School of Materials Science and Engineering, Changchun University of Science and Technology, Changchun 130022, China

*Email: xbzhang@ciac.ac.cn
} 


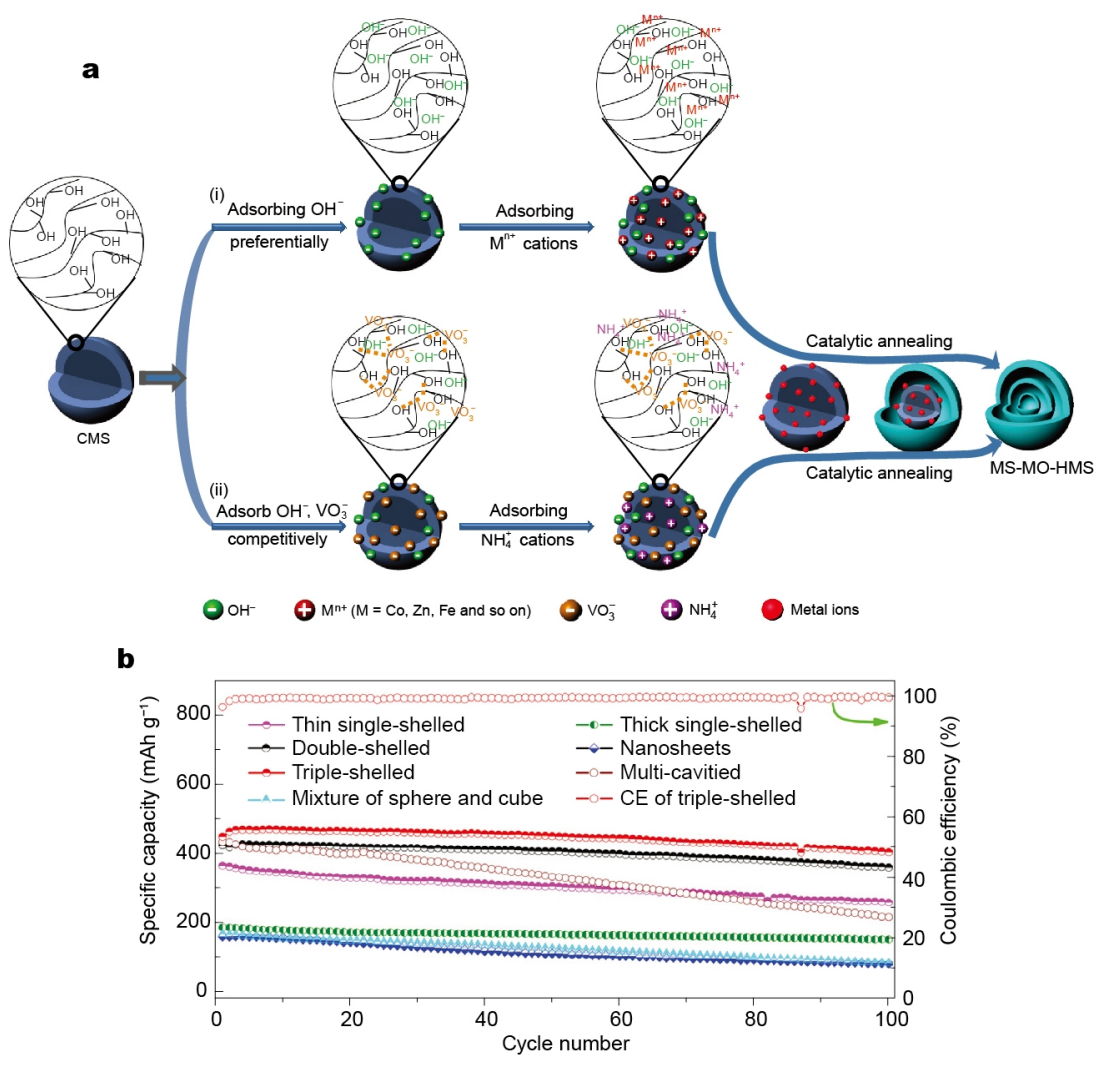

Figure 1 Multi-shelled $\mathrm{V}_{2} \mathrm{O}_{5}$ hollow microspheres. (a) The synthesis routes of multi-shelled hollow microspheres: (i) the conventional cation-adsorption process and (ii) the reported anion-adsorption process. (b) Discharge capacity versus cycle number for the prepared $\mathrm{V}_{2} \mathrm{O}_{5}$ hollow microspheres and nanosheets at $1000 \mathrm{~mA} \mathrm{~g}^{-1}$. Reprinted with permission from Ref. [8], Copyright 2016, Nature Publishing Group.

advanced electrode materials for next generation LIBs.

Corresponding authors Prof. Ranbo Yu from University of Science and Technology Beijing, Prof. Yu Zhang of Beihang University and Prof. Dan Wang of the Institute of Process Engineering, Chinese Academy of Sciences noted that though the conventional cation-adsorption approach has been adopted in previous studies, the underlying mechanism has received little attention. Thus, the modelling and calculations were carried out to support their hypothesis about the mechanism of anion adsorption strategy. Moreover, using this general strategy, some other metal oxides (MO) $\left(\mathrm{MnO}_{2}, \mathrm{MoO}_{3}, \mathrm{Cr}_{2} \mathrm{O}_{3}\right.$ and $\left.\mathrm{WO}_{3}\right)$ of $\mathrm{M}$-HMs with a well-controlled shell number, thickness, porosity and crystallinity have also been obtained, thus greatly enriching the family of M-MO-HMs. Most importantly, this revolutionary work endows $\mathrm{V}_{2} \mathrm{O}_{5}$ cathode material with high performance and makes it tremendous potential for applications in the next generation of high-performance LIBs.

Received 6 July 2016; accepted 6 July 2016; published online 26 July 2016
1 Tarascon JM, Armand M. Issues and challenges facing rechargeable lithium batteries. Nature, 2001, 414: 359-367

2 Gao XP, Yang HX. Multi-electron reaction materials for high energy density batteries. Energy Environ Sci, 2010, 3: 174-189

3 Pan A, Wu HB, Yu L, et al. Template-free synthesis of $\mathrm{VO}_{2}$ hollow microspheres with various interiors and their conversion into $\mathrm{V}_{2} \mathrm{O}_{5}$ for lithium-ion batteries. Angew Chem Int Ed, 2013, 52: 2226-2230

4 Hu YS, Liu X, Müller JO, et al. Synthesis and electrode performance of nanostructured $\mathrm{V}_{2} \mathrm{O}_{5}$ by using a carbon tube-in-tube as a nanoreactor and an efficient mixed-conducting network. Angew Chem Int Ed, 2009, 48: 210-214

5 Wang $\mathrm{H}$, Ma D, Huang $\mathrm{Y}$, et al. Electrospun $\mathrm{V}_{2} \mathrm{O}_{5}$ nanostructures with controllable morphology as high-performance cathode materials for lithium-ion batteries. Chem Eur J, 2012, 18: 8987-8993

6 Tarascon JM, Poizot P, Laruelle S, et al. Nano-sized transitionmetal oxides as negative-electrode materials for lithium-ion batteries. Nature, 2000, 407: 496-499

7 Wang HG, Yuan S, Ma DL, et al. Electrospun materials for lithium and sodium rechargeable batteries: from structure evolution to electrochemical performance. Energy Environ Sci, 2015, 8: $1660-1681$

8 Wang J, Tang H, Zhang L, et al. Multi-shelled metal oxides prepared via an anion-adsorption mechanism for lithium-ion batteries. Nat Energy, 2016, 1: 16050 